\title{
Hypothermic circulatory arrest for aortic dissection with cryoglobulinemia
}

\author{
Kazuhiro Yamazaki $^{1}$, Kenji Minatoya ${ }^{2}$, Kazuhisa Sakamoto ${ }^{1}$, Koji Kitagori ${ }^{3}$, Masanori \\ Okuda $^{3}$, Hideo Kanemitsu ${ }^{1}$, Takahide Takeda ${ }^{1}$, Masahide Kawatou ${ }^{1}$, Jiro Sakai ${ }^{1}$, Motoyuki \\ Kumagai $^{4}$, Takashi Tsuji ${ }^{1}$, Kosaku Murakami ${ }^{5}$, Tsuneyasu Yoshida ${ }^{5}$, Yujiro Ide ${ }^{1}$, and \\ Tadashi Ikeda ${ }^{1}$ \\ ${ }^{1}$ Graduate School of Medicine, Kyoto University \\ ${ }^{2}$ Natl Cerebral \\ ${ }^{3}$ Affiliation not available \\ ${ }^{4}$ Kyoto University Graduate School of Medicine Department of Cardiovascular Surgery \\ ${ }^{5}$ Graduate of School of Medicine, Kyoto University
}

June 13, 2020

\begin{abstract}
Cryoglobulinemia is a cold-reactive autoimmune disease. A 64-year-old man with active cryoglobulinemia presented Stanford type A acute aortic dissection. He had been treated with immunosuppressive drugs and plasma exchange (PE) at our hospital; subsequently, qualitative analysis of cryogobulin (CG) was negative. He underwent emergency ascending aorta replacement using cardiopulmonary bypass (CPB) under deep hypothermia circulatory arrest with selective cerebral perfusion. The total CPB time, aortic cross clamp time, and selective cerebral perfusion time were 255, 153, 56 minutes, respectively, and the minimal nasopharyngeal temperature was $17.3^{\circ} \mathrm{C}$. Our patient had no significant perioperative complications. Hence, if PE is performed appropriately and CG is negative, patients with cryoglobulinemia who exhibit severe preoperative symptoms can safely undergo surgery with deep hypothermia.
\end{abstract}

\section{Introduction}

Cryoglobulinemia is a clinical disorder characterized by the presence of cryogobulins (CGs) in the serum. CGs are immunoglobulins (IGs) that precipitate in vitro at temperatures below the normal body temperature $\left(<37^{\circ} \mathrm{C}\right)$ and redissolve upon warming. Cardiovascular surgeries, such as cardiopulmonary bypass (CPB) usually requires systemic hypothermia, has a risk of organ disorders due to microangiopathy induced a rapid increase in blood viscosity. We present a case of ascending aorta replacement with CPB under deep hypothermic circulatory arrest in a patient who experienced acute aortic dissection during cryoglobulinemia treatment.

\section{Case report}

A 64-year-old man was referred to our hospital 6 months ago for cryoglobulinemia. He had previously been treated for gangrene of the right toe 2 years prior during winter; however, since then symptoms of necrosis and numbness of the lower extremities worsened. CGs were detected through qualitative analysis (Fig. 1a). However, no additional abnormalities were detected upon further examination. Hence, he was diagnosed with active idiopathic cryoglobulinemia. Subsequently, immunosuppressive therapy with corticosteroids and cyclophosphamide was initiated. However, he had persistent numbness in the lower limbs, and CG levels slightly decreased (Fig. 1b) without a negative outcome. Therefore, he underwent three rounds of plasma 
exchange (PE) and double filtration plasmapheresis; subsequently, qualitative analysis of CG was found to be negative (Fig. 1c). The day after PE, our patient experienced sudden chest and back pain. Contrast-enhanced computed tomography revealed Stanford type A acute aortic dissection.

Regarding the use of hypothermia during surgery, since the patient had undergone PE the day before, we decided to perform the surgery using CPB under deep hypothermia circulatory arrest. After esophageal temperature was lowered to $20^{\circ} \mathrm{C}$ and circulatory arrest was accomplished, selective cerebral perfusion was initiated. Cardiac arrest was induced using retrograde blood cardioplegia at $20^{\circ} \mathrm{C}$, which was administered every 30 minutes. We finished anastomosing the distal aorta, and then, started recirculation and rewarming. After anastomosis of the proximal aorta, antegrade blood cardioplegia was administered at $30^{\circ} \mathrm{C}$ to $35^{\circ} \mathrm{C}$. Weaning from $\mathrm{CPB}$ was smooth and uneventful, with no changes in cardiac function. Also, no signs of agglutinin reaction and thrombosis in the $\mathrm{CPB}$ circuit were observed. The total $\mathrm{CPB}$ time, aortic cross clamp time, and selective cerebral perfusion time were 255,153 , and 56 minutes, respectively. The minimal nasopharyngeal temperature during the procedure was $17.3^{\circ} \mathrm{C}$.

One day after surgery, the patient was extubated in the intensive care unit and had no significant postoperative complications. Six months after surgery, he received outpatient treatment for cryoglobulinemia.

\section{Discussion}

CGs are proteins that become insoluble and typically precipitate at temperatures below normal body temperature and dissolve back into the serum upon rewarming CGs can be either IGs or a mixture of IGs and complement components ${ }^{1}$. In open heart surgery, systemic hypothermia, and cold or tepid cardioplegia procedure using $\mathrm{CPB}$ are typically used to protect the myocardium and internal organs. However, in patients with cryoglobulinemia, these methods may have deleterious effects. Regrettably, there are no guidelines for the management of patients with CG during CPB. Therefore, we searched an extensive literature and found only13 cases of $\mathrm{CPB}$ in patients with cryoglobulinemia.

The profiles and treatment of the 14 cases, including our case, are presented in Table 1 and 2. The severity of preoperative CG was based on various conditions. Among these patients, especially, our patient had very severe symptoms and needed intensive treatment for cryoglobulinemia (Table 1).

In many cases, surgery was performed using systemic normothermia $\left(>35^{\circ} \mathrm{C}\right)$ (Table 2$)$. However, since Stanford type A dissection may require total arch replacement, we had to lower the body temperature of our patient. The literature review revealed thar only 3 cases have been reported which used moderate hypothermia $\left(25^{\sim} 27^{\circ} \mathrm{C}\right)$, two of them underwent PE previously for decreasing the CG levels before surgery 2,3 .

In cardioplegia, warm cardioplegia via antegrade was typically used, and two of 3 cases used moderate hypothermia were not used blood cardioplegia ${ }^{2,3}$. In our case, myocardial protection was performed with intermittent retrograde cold blood cardioplegia during hypothermia. During these procedures, there may be a risk of thrombosis of the coronary veins. Hence, after proximal anastomosis, the temperature was increased approximate $35^{\circ} \mathrm{C}$ and antegrade warm cardioplegia was initiated for flushing thrombus.

Fortunately, in our case, the qualitative analysis result was negative for CG after PE therapy. Hence, surgery could be performed under deep hypothermia and cold cardioplegia, even for the patient with severe cryoglobulinemia symptoms.

\section{Conclusions}

We successfully performed aortic replacement under hypothermic circulation arrest in a patient with acute aortic dissection during cryoglobulinemia treatment and obtained favorable results. Our patient underwent $\mathrm{PE}$ a day before $\mathrm{CPB}$ and was negative for CG. Thus, if PE is performed appropriately and CG is negative, then CPB under hypothermia can be considered in patients with cryoglobulinemia who exhibit severe preoperative symptoms.

Conflicts of interest. The authors declare that they have no competing interests. 
Authors' contributions . All authors contributed to the writing of the final manuscript.

\section{REFERENCES}

1. Ostojic P, Jeremic IR. Managing refractory cryoglobulinemic vasculitis: challenges and solutions. J Inflamm Res 2017;10:49-54.

2. Kotsuka Y, Nakajima J, Miyairi T, Nakahara H, Suzuki M, Kanda J, et al. Coronary artery bypass grafting in a patient with cryoglobulinemia. J Cardiovasc Surg (torino) 1991;32:53-55.

3. Osada T, Kawachi K, Uchino T, Hirayama T, Ishimaru S, Furukawa K. A successful case of thoracic aortic aneurysm with mixed cryoglobulinemia. Nihon Kyobu Geka Gakkai Zasshi. 1992;40:1100-1104.

4. Muehreke DD, Torchiana DF. Warm heart surgery in patients with cold autoimmune disorder. AnnThorac Surg 1993;55:532-533.

5. Murata S, Tabayashi K, Schinozaki S, Shimizu M, Ito T, Konnai T. Successful cardiac surgery using normothermic cardiopulmonary bypass in an elderly patient with cryoglobulinemia. Nihon Kyobu Geka Gakkai Zasshi 1995;43:1100-1104.

6. Satoh S, Satoh D, Kaise A, Hashimoto Y. Anethetic management of a patient with cryoglobulinemia for open-heart surgery under normothermic cardiopulmonary bypass. J Jpn Soci Clin Aneth1995;15:649653.

7. Yatsu Y, Kimura F, Muraoka M, Tsubo T, Ishihara H, Matsuki, A. Perioperative management of a patient with cryoglobulinemia for open heart surgery. Masui 1998;47:53-56.

8. Fontana M, Ruchat P, Horisberger J, Aubert V, Mayor C, Spertini F. Prevention of cryoprecipitation during cardiopulmonary bypass in a patient with HIV-HCV co-infections. Perfusion 2006;21:263-265.

9. Hida M, Ishisone A, Sato K, Yamada M, Yamashita Y, Otake J, et al. Extra corporeal circulation for a patient with cryoglobulinemia. Jpn J Extra-Corporeal Tecnology 2011;38:439-441 (in Japanese).

10. Nasu T, Watanabe S, Chiba F, Tamura T, Suzuki R, Tsuji Y, et al. A case of pulmonary endarterectomy using normothermic cardiopulmonary bypass for a patient with cryoglobulinemia. J HSCET 2014;24:3234 (in Japanese).

11. Fakih HAM, Elueze E, Vij R. Coronary Artery Bypass Grafting in a Patient with Active idiopathic cryoglobulinemia: revisiting the issue. J Community Hosp Intern Med Perspect 2016;6 :30351 doi: 10.3402/jchimp.v6.30351.

12. Satomi S, Kasai A, Hamaguchi E, Tsutsumi YM, Tanaka K. Normothermic cardiopulmonary bypass in patient with Waldenstrm's macroglobulinemia and cryoglobulinemia: a case report. A Case Rep 2017;9:162-163.

13. Okamoto T, Kimura T, Kume M. Severe aortic stenosis in a patient with cryoglobulinemia. J Jpn Surg Assoc. 2017;78:1248-1251.

14. Vela RJ, Krueger A, Huffman LC, Bajona P. Cryoglobulinemia: caution for patients undergoing cardiac surgery. Asian Cardiovasc Thorac Ann 2019;27:600-602.

\section{Figure legends}

Qualitative analysis of cryoglobulin (CG). (a) Upon admission to our hospital and (b) during immunosuppressive treatment with corticosteroids and cyclophosphamide, CG levels slightly decreased without a negative outcome. (c) After three rounds of plasma exchange (PE) and double filtration plasmapheresis. CG was found to be negative.

\section{Hosted file}

JCS \begin } \{ \text { CJK\} \{UTF8\}\{gbsn\} \end\{CJK\}\selectlanguage\{english\}TABLES.docx available at } https://authorea.com/users/332602/articles/459031-hypothermic-circulatory-arrest-foraortic-dissection-with-cryoglobulinemia 


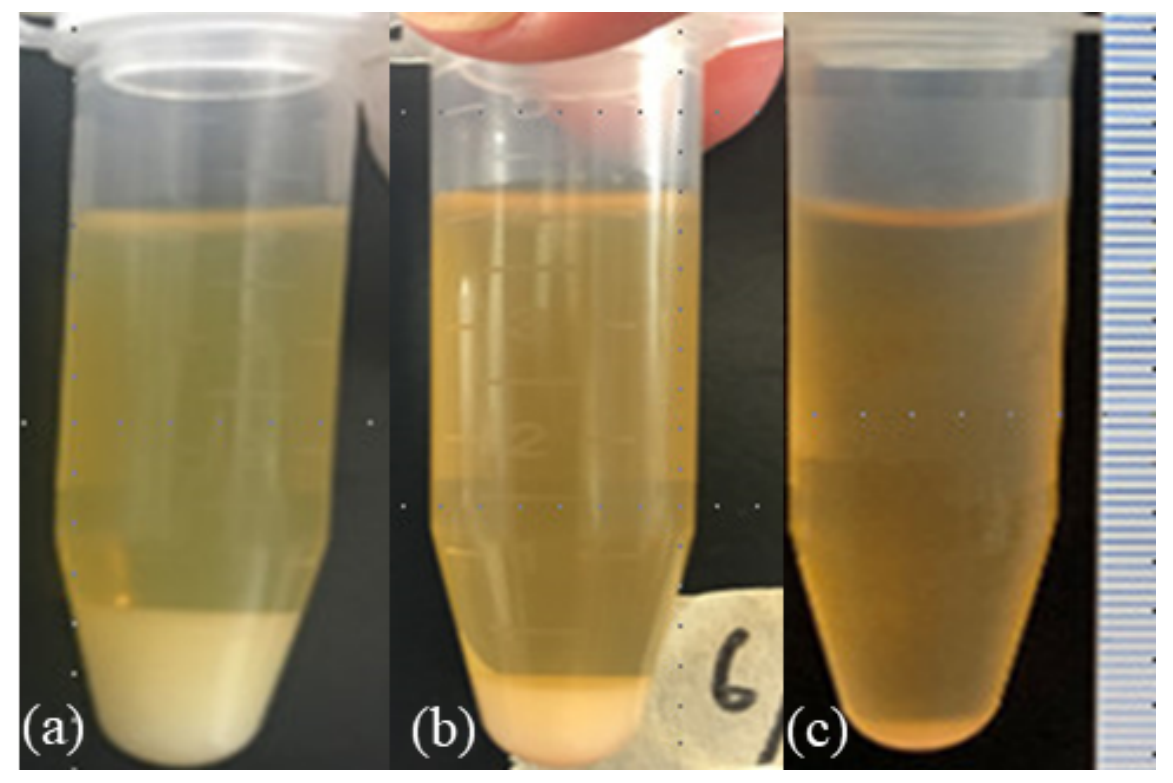

